\title{
Diverse types of review studies based on their approach to retrieving and summarizing original findings
}

\author{
Rezaeian M, PhD \\ - Professor, Epidemiologist, Social Medicine Department, Occupational Environmental Research Center, Rafsanjan \\ Medical School, Rafsanjan University of Medical Sciences, Rafsanjan, Iran.
}

Abstract

Received: April 2014, Accepted: April 2015

Background: We are living in an era in which different branches of science are growing very rapidly. Therefore, retrieving and summarizing all new valid findings on a specific subject is one of the most important priorities of scientists. The aim of the present article is to categorize different review studies within the health domain based on their approach to retrieving and summarizing original findings.

Methods: The most relevant articles have been selected and summarized in order to accomplish the aim of the study.

Results: Narrative reviews, systematic reviews, meta-analyses from published data, and individual participant data meta-analyses are different types of review studies. More recently, prospectively planned pooled analyses have also been introduced.

Conclusions: Diverse types of review studies have been developed one by one over the course of time based on their approaches to retrieving and summarizing original findings. Therefore, they have their own especial applications and scientists of different disciplines need to know their advantages and disadvantages.

Keywords: Narrative Reviews, Systematic Review, Meta-Analysis.

\section{Introduction}

We are living in an era in which different branches of sciences are growing rapidly. Therefore, retrieving and summarizing all new valid findings on a specific subject is one of the most important priorities of scientists. Review studies with the aim of retrieving and summarizing original findings in a specific field are an important reflection of the scientific community in response to this growing demand.

However, in order to obtain better results from review studies, the method of carrying out such studies has substantially changed over time (1-9). These substantial changes mostly occur in a review study's method of retrieving and summarizing original articles in a specific domain. The aim of the present article is to categorize different review studies based on their approach to retrieving and summarizing original articles.

\section{Methods}

The most relevant articles have been selected and summarized in order to accomplish the aim of the study.

\section{Results}

Narrative reviews, systematic reviews, metaanalyses from published data, and individual participant data meta-analyses are different

\footnotetext{
* Corresponding author: Mohsen Rezaeian, School of Medicine, Occupational Environmental Reseaech Center, Rafsanjan University of Medical Sciences, Rafsanjan, Iran.

Email: moeygmr2@yahoo.co.uk
} 
types of review studies. More recently, prospectively planned pooled analyses have also been introduced. In what follows, these diverse types of studies plus their advantages and disadvantages will be discussed.

\section{Narrative or traditional review}

The first and most well-known reviews are narrative or traditional reviews in which usually an expert in a given subject retrieves and summarizes original articles.

In this traditional format, the reviewers usually do not specify their strategy of search. In other words, reviewers do not give the details of their literature review, and as a result, other people are not able to follow their search strategy and produce the same results $(10,11)$.

This could be referred to as the first Achilles' heel of the traditional reviews and has caused a number of criticisms. The second Achilles' heel of a traditional review is that it only produces a qualitative and not a quantitative result.

In order to tackle the first shortcoming of narrative reviews, the scientific community invented the second type of review which is called systematic review.

\section{Systematic review}

Contrary to narrative review, a reviewer in a systematic review gives sufficient details of his/her search strategy in order to make a systematic review able to be scrutinized precisely and be reproducible by other scientists (12-15).

Therefore, in materials and methods of a systematic review, the search strategy (i.e., search engines, keywords, and time frame), hand searching, and exclusion and inclusion criteria set for selecting the original articles are explained in detail. Such a detailed search strategy aims at removing the first shortcoming of the traditional reviews and makes the results of a systematic review less biased and more valid and reliable.

However, still some argue that the results of a systematic review, like a traditional review, are qualitative. We need to quantify the results to better inform others about the latest scientific findings (e.g., in what order, and how much the exposure changes the outcome). This led the scientific community to inventing the third type of review, which is called meta-analysis from published data.

\section{Meta-analysis from published data}

Meta-analysis from published data was invented to tackle the qualitative summarizing of traditional and systematic reviews. The chief aim of such studies, therefore, is to produce a quantitative pooled estimate by applying statistical methods (16, 17).

The method of retrieving original papers in a meta-analysis is like that of a systematic review. However, in a meta-analysis, the investigator will try to produce a figure with its confidence interval to quantify the relation between the exposure and the outcome.

Nevertheless, meta-analysis from published data also has some weaknesses. For instance, it might be possible that the retrieved papers only consist of those original papers that found a significant relation between the exposure and outcome. This is referred to as publication bias and might jeopardize meta-analyses as well as systematic and traditional reviews. However, there are statistical methods, such as funnel plot, which help investigators to determine the possibility, and also, the extent of the publication bias $(18,19)$.

There is another important Achilles' heel for meta-analysis the extent of which depends 
on the amount of the heterogeneity between the original retrieved studies.

Usually, original studies apply different methods to quantify the relation between the exposure and outcome. Similarly, they might investigate the relation between exposure and outcome within different settings, and diverse age and sex groups. If there are heterogeneities between original studies, then, producing a quantitative pooled estimate would not be suggested.

\section{Individual participant data meta- analysis}

Individual participant data meta-analysis was invented to tackle some of the shortcomings of meta-analysis from published data. This method is ideal for removing heterogeneities between original studies (20-24). In this type of review, the technique of retrieving original papers is similar to that of systematic review and meta-analysis from published data.

However, after selecting the original papers, the investigators contact the corresponding author of each original retrieved paper to introduce themselves and explain their plan of study, and then, ask for raw data. The aim of this type of meta-analysis is to collect raw data from original studies, and then, to combine those raw data in a way to minimize the possibility of any heterogeneities.

By doing this the investigators are not only able to decrease the possibility of any heterogeneity between individual studies, but also to increase the power of their study in order to detect any significant relations between the exposure and the outcome. However, the extent of success in such meta-analyses is related to the quality of raw data of each original study.

\section{Prospectively planned pooled analysis}

In practice, individual participant data metaanalysis would be very difficult to implement (25). Therefore, scientists invented a new type of study which is called prospectively planned pooled analysis (2629).

In this new type of investigation, which is not a review study, high-quality original studies with similar design and reporting guidelines are carried out in different communities. The chief aim of such a design is to minimize the heterogeneities between studies. As a result, it is hoped that prospectively planned pooled analysis will help alleviate many problems of individual participant data meta-analyses.

\section{Publication guidelines for diverse types of review studies}

It is worth mentioning that there are, at the very least, two main publication guidelines for diverse types of review studies. The first guideline which is called the Quality of Reporting of Meta-analyses (QUOROM Statement) has been released in 1996. This guideline has primarily focused on the reporting of meta-analyses of randomized controlled trials (30).

The second guideline which is called the Preferred Reporting Items for Systematic Reviews and Meta-analyses (PRISMA Statement) is a revision of the QUOROM Statement and has been released in 2009. This guideline has addressed several theoretical and practical progresses in the science of systematic reviews (31).

Publication guidelines have been developed to help researchers for optimal reporting of diverse types of studies. Nevertheless, my personal experiences indicate that it is better to extend the application of such publication guidelines to cover the designing stage and protocol writing of each relevant study (32). 


\section{Concluding remarks}

Diverse types of review studies have been developed one by one over time based on their approaches to retrieving and summarizing original findings. Therefore, they have their own especial applications and scientists of different disciplines need to know their advantages and disadvantages.

Conflict of interest: Non declared.

\section{References}

1. Blettner M, Sauerbrei W, Schlehofer B, Scheuchenpflug T, Friedenreich C. Traditional reviews, meta-analyses and pooled analyses in epidemiology. Int $\mathbf{J}$ Epidemiol 1999; 28(1):1-9.

2. Parker M, Gillespie L, Gillespie W, Handoll $\mathrm{H}$, Madhok R, Morton L; Cochrane Collaboration Musculoskeletal Injuries Collaborative Review Group. Systematic reviews, meta-analyses, and methodology. J Bone Joint Surg Am 2001; 83-A(9):1433-5.

3. Montori VM, Swiontkowski MF, Cook DJ. Methodologic issues in systematic reviews and meta-analyses. Clin Orthop Relat Res 2003; (413):43-54.

4. Shrier I, Boivin JF, Platt RW, Steele RJ, Brophy JM, Carnevale F, et al. The interpretation of systematic reviews with meta-analyses: an objective or subjective process? BMC Med Inform Decis Mak 2008; 8:19.

5. Ressing M, Blettner M, Klug SJ. Systematic literature reviews and meta-analyses: part 6 of a series on evaluation of scientific publications. Dtsch Arztebl Int. 2009; 106(27):456-63.

6. Ryś P, Władysiuk M, Skrzekowska-Baran I, Małecki MT. Review articles, systematic reviews and meta-analyses: which can be trusted? Pol Arch Med Wewn 2009; 119(3):148-56.

7. Leucht S, Kissling W, Davis JM. How to read and understand and use systematic reviews and meta-analyses. Acta Psychiatr Scand 2009; 119(6):443-50.

8. Noordzij M, Hooft L, Dekker FW, Zoccali C, Jager KJ. Systematic reviews and metaanalyses: when they are useful and when to be careful. Kidney Int 2009; 76(11):1130-6.

9. Noordzij M, Zoccali C, Dekker FW, Jager KJ. Adding up the evidence: systematic reviews and meta-analyses. Nephron Clin Pract 2011; 119(4):c310-6.

10. Green BN, Johnson CD, Adams A. Writing narrative literature reviews for peerreviewed journals: secrets of the trade. J Chiropr Med 2006; 5(3):101-17.

11. Murphy CM. Writing an effective review article. J Med Toxicol 2012; 8(2):89-90.

12. Bettany-Saltikov J. Learning how to undertake a systematic review: part 1 . Nurs Stand. 2010; 24(50):47-55; quiz 56.

13. Bettany-Saltikov J. Learning how to undertake a systematic review: part 2. Nurs Stand 2010; 24(51):47-56; quiz 58, 60.

14. Ng KH, Peh WC. Writing a systematic review. Singapore Med J 2010; 51(5):362-6.

15. Wright RW, Brand RA, Dunn W, Spindler KP. How to write a systematic review. Clin Orthop Relat Res 2007; 455:23-9.

16. Jain V, Sharma R, Singh S. Doing metaanalysis in research: a systematic approach. Indian J Dermatol Venereol Leprol 2012; 78(3):242-50.

17. Rudnicka AR, Owen CG. An introduction to systematic reviews and meta-analyses in health care. Ophthalmic Physiol Opt 2012; 32(3):174-83.

18. Copas J, Shi JQ. Meta-analysis, funnel plots and sensitivity analysis. Biostatistics. 2000; 1(3):247-62.

19. Sterne JA, Egger M. Funnel plots for detecting bias in meta-analysis: guidelines on choice of axis. J Clin Epidemiol 2001; 54(10):1046-55.

20. Riley RD, Lambert PC, Abo-Zaid G. Metaanalysis of individual participant data: rationale, conduct, and reporting. BMJ. 2010; 340:c221.

21. Abo-Zaid G, Sauerbrei W, Riley RD. Individual participant data meta-analysis of prognostic factor studies: state of the art? BMC Med Res Methodol 2012; 12:56.

22. Clarke MJ. Individual patient data metaanalyses. Best Pract Res Clin Obstet Gynaecol 2005; 19(1):47-55.

23. Fisher DJ, Copas AJ, Tierney JF, Parmar MK. A critical review of methods for the assessment of patient-level interactions in individual participant data meta-analysis of randomized trials, and guidance for practitioners. J Clin Epidemiol 2011; 64(9):949-67.

24. Fibrinogen Studies Collaboration, Jackson D, White I, Kostis JB, Wilson AC, Folsom $\mathrm{AR}$, et al. Systematically missing confounders in individual participant data meta-analysis of observational cohort studies. Stat Med 2009; 28(8):1218-37.

25. Ahmed I, Sutton AJ, Riley RD. Assessment of publication bias, selection bias, and unavailable data in meta-analyses using 
individual participant data: a database survey. BMJ $2012 ; 344: d 7762$.

26. Altman DG, Riley RD. Primer: an evidencebased approach to prognostic markers. Nat Clin Pract Oncol 2005; 2(9):466-72.

27. Riley RD, Sauerbrei W, Altman DG. Prognostic markers in cancer: the evolution of evidence from single studies to metaanalysis, and beyond. Br J Cancer 2009; 100(8):1219-29.

28. Stein DJ, Bandelow B, Merideth C, Olausson B, Szamosi J, Eriksson H. Efficacy and tolerability of extended release quetiapine fumarate (quetiapine $\mathrm{XR}$ ) monotherapy in patients with generalised anxiety disorder: an analysis of pooled data from three 8-week placebo-controlled studies. Hum Psychopharmacol 2011; 26(8):614-28.

29. Weisler RH, Montgomery SA, Earley WR, Szamosi J, Lazarus A. Efficacy of extended release quetiapine fumarate monotherapy in patients with major depressive disorder: a pooled analysis of two 6-week, doubleblind, placebo-controlled studies. Int Clin Psychopharmacol 2012; 27(1):27-39.

30. Moher D, Cook DJ, Eastwood S, Olkin I, Rennie D, Stroup DF. Improving the quality of reports of meta-analyses of randomised controlled trials: the QUOROM statement. QUOROM Group. Br J Surg 2000; 87(11):1448-54.

31. Moher D, Liberati A, Tetzlaff J, Altman DG; PRISMA Group. Preferred reporting items for systematic reviews and metaanalyses: the PRISMA Statement. Ann Intern Med 2009; 3(3):e123-30.

32. Rezaeian M. The application of publication guidelines should extend to cover their designing stage and protocol writing. Ann Epidemiol 2013; 23(12):815. 